\title{
ANXA2 Silencing Inhibits Proliferation, Invasion, and Migration in Gastric Cancer Cells
}

\author{
Rui Xie, ${ }^{1}$ Jia Liu, ${ }^{1}$ Xuefeng Yu, ${ }^{1}$ Chunfeng Li, ${ }^{1}$ Yufeng Wang, ${ }^{1}$ Wei Yang, \\ Jiahe Hu, ${ }^{1}$ Ping Liu, ${ }^{1}$ Hong Sui, ${ }^{1}$ Peiqiang Liang, ${ }^{2}$ Xinyan Huang, ${ }^{2}$ Lijuan Wang, \\ Yuxian Bai, ${ }^{1}$ Yingwei Xue $\mathbb{D}^{1},{ }^{1}$ Jiuxin Zhu $\mathbb{D}^{2},{ }^{2}$ and Tianyi Fang $\mathbb{D}^{1}$ \\ ${ }^{1}$ Harbin Medical University Cancer Hospital, 150 Haping Road, Harbin 150081, China \\ ${ }^{2}$ Department of Pharmacology (State-Province Key Laboratories of Biomedicine-Pharmaceutics of China, Key Laboratory of \\ Cardiovascular Medicine Research, Ministry of Education), College of Pharmacy, Harbin Medical University, Harbin 150081, China
}

Correspondence should be addressed to Jiuxin Zhu; zhujiuxin@hrbmu.edu.cn and Tianyi Fang; fangtianyi@hrbmu.edu.cn

Received 29 November 2018; Revised 15 March 2019; Accepted 28 March 2019; Published 2 May 2019

Academic Editor: Thomas E. Adrian

Copyright (c) 2019 Rui Xie et al. This is an open access article distributed under the Creative Commons Attribution License, which permits unrestricted use, distribution, and reproduction in any medium, provided the original work is properly cited.

\begin{abstract}
Annexin A2 (ANXA2) has been well known to associate with the progress of malignant tumor. However, the biological behavior of ANXA2 in gastric cancer (GC) remains unclear. We made a hypothesis in transcriptome level from TCGA datasets. Then, we used immunohistochemical staining to quantify the expression level of ANXA2 protein in GC tissues compared with adjacent tissues. Quantitative real-time PCR and western blot were used for analyzing ANXA2 expression in human GC (SGC-7901, MKN45, BGC-823, and AGS) cell lines. We investigated the effect of a lentivirus-mediated knock-down of ANXA2 on the proliferation, invasion and migration of gastric cancer AGS cells. Cell proliferation was examined by MTT and colony formation tests. Cell apoptosis and cycle were measured by flow cytometry. Migration and invasion were detected by transwell assay. We found that high expression of ANXA2 can increase the mobility of cancer cells from TCGA datasets. ANXA2 was upregulated in GC tissues compared with adjacent tissues. AGS cell line displayed significantly higher expression of ANXA2 among the four GC cell lines. In addition, ANXA2 silencing led to a weakened ability of proliferation, invasion, and migration in GC cells; targeting of ANXA2 may be a potential therapeutic strategy for GC patients.
\end{abstract}

\section{Introduction}

Gastric cancer (GC) is the fifth highest incidence malignant tumor in the world and the third dominant cause of cancer death, with a 5-year survival rate of only $20 \%$ to $25 \%$ worldwide $[1,2]$. Despite the improvement of therapeutic methods with surgical resection, chemotherapy, radiotherapy, immunotherapeutic strategies, and targeted therapies, invasion and metastasis lead to the poor prognosis of GC patients and have become a significant clinical challenge [3-7]. Therefore, finding new molecular markers which are related to metastasis and poor outcome may contribute to affording new insights into diagnostic decision and novel therapies for GC patients.

ANXA2 is a $36 \mathrm{kDa}$ calcium-dependent phospholipidbinding cytoskeletal protein; it is also named as Annexin II, Annexin a2, p36, and lipocortin II [8]. Upregulation of ANXA2 was observed in many different cancer types, including hepatoma [9], pancreatic cancer [10], breast cancer [11], glioma [12], colorectal cancer [13], and GC [14]. ANXA2 mainly participates in cell membranes formation and takes effect on regulate cytoskeleton. The cytoskeleton changes are common in malignant transformation, adhesion, movement, and metastasis which may promote tumor cell to move [15]. Thus, targeting cancerous cells motility may be important to the treatments. However, it is difficult to investigate the exact function of ANXA2 in GC directly. With the development of transcriptomics, analysis of transcriptome sequencing data from real pathological specimens may provide a comprehensive background for us to understand the relationship between ANAX2 and GC cells.

To clarify this phenomenon, we analyzed the ANAX2 expression in GC tissues from The Cancer Genome Atlas (TCGA) database by R2 analysis platform. Gene Correlation 
analysis, Gene Ontology (GO) analysis, and Kyoto Encyclopedia of Genes and Genomes (KEGG) analysis were performed at the level of transcriptomics. The expression level of ANXA2 protein in GC tissues compared with adjacent tissues was evaluated. Then, we focused on the motility changes of GC cells when inhibiting ANXA2, including the function of proliferation, invasion, and migration.

\section{Materials and Methods}

2.1. Bioinformatics Analysis. The RNA sequencing (RNA-seq) data was downloaded from TCGA database (https://portal.gdc.cancer.gov/); it contained ANXA2 RNA expression data for human GC profiles, including 415 tissue samples. Gene Correlation analysis was performed with the R2: Genomics Analysis and Visualization Platform (http://r2.amc.nl); the genes and pathways associated with ANXA2 were tested by GO and KEGG pathway analysis. Correlation statistics were calculated using the R2 platform and $P<0.05$ was statistically significant.

2.2. Tissue Microarray. A GC tissue microarray (HStmAde180Sur-06) was obtained from Shanghai Outdo Biotech. GC was staged according to the WHO classification criteria: 90 tumor tissues with stages I to IV and paired noncancerous tissues. The present study was approved by the Institutional Review Boards of participating institutions.

2.3. Immunohistochemistry. Immunohistochemical staining for ANXA2 was performed by using the EnVision Detection Systems (Dako, Glostrup, Denmark) according to the manufacturer's instructions, with ANXA2 rabbit monoclonal antibody (Cell Signaling Technology, Beverly, MA, USA) at 1:2000 dilution as the primary antibody. Staining intensity of ANXA2 was graded as follows: negative staining $(-),<5 \%$ of total cells; weak staining $(+), 5 \%-15 \%$; moderate staining $(++), 16 \%-50 \%$; and diffuse positive staining $(+++),>50 \%$.

2.4. Cell Culture. GC cell lines including SGC-7901, MKN45, BGC-823, and AGS cells with different grade of differentiation were cultured in RPMI1640 medium supplemented with $10 \%$ fetal bovine serum, $100 \mathrm{U} / \mathrm{ml}$ penicillin, and 0.1 $\mathrm{mg} / \mathrm{ml}$ streptomycin, in humidified air containing 5\% CO2 at $37^{\circ} \mathrm{C}$. The culture medium was replaced every other day.

2.5. RNA Isolation and Reverse Transcription. Total RNA was extracted from cells using TRIzol Reagent (Invitrogen, Carlsbad, CA, USA) according to manufacturer's protocol. RNA concentration and quality were determined by the NanoDrop 2000c Spectrophotometer (Thermo Scientific, MA, USA). Subsequently, cDNA was synthesized using M-MLV reverse transcriptase, dNTPs, RNase inhibitor (Promega, Madison, WI, USA), and an oligo dT purchased from Sangon Biotech (Shanghai, China).

2.6. Quantitative PCR ( $q$-PCR). Then, q-PCR was carried out using SYBR Master Mixture (TaKaRa, Ohtsu, Japan) and TaKaRa Thermal Cycler Dice Real-Time PCR System TP800.
The primer sequences were as follows: $5^{\prime}$-GTGGTGGAGATGACTGAAGCC-3' (sense) and $5^{\prime}$-CCACGGGGACTGTTATTCG-3' (antisense) were for ANXA2 (110 bp). Glyceraldehyde 3-phosphate dehydrogenase (GAPDH) was used as an internal control; 5'-TGACTTCAACAGCGACACCCA- $3^{\prime}$ (sense) and $5^{\prime}$-CACCCTGTTGCTGTAGCCAAA$3^{\prime}$ (antisense) were for GAPDH (121 bp). The thermal cycling conditions consisted of 1 cycle at $95^{\circ} \mathrm{C}$ for $30 \mathrm{~s}$, followed by 45 cycles at $95^{\circ} \mathrm{C}$ for $5 \mathrm{~s}$ and $60^{\circ} \mathrm{C}$ for $30 \mathrm{~s}$. Data were analyzed by the comparative threshold $(2-\Delta \Delta \mathrm{Ct})$ method.

2.7. Western Blot. Proteins in cell lysates were separated using sodium dodecyl sulfate-polyacrylamide gel electrophoresis and electrophoretically transferred to polyvinylidene difluoride membranes. Proteins were probed overnight at $4^{\circ} \mathrm{C}$ with ANXA2 rabbit monoclonal antibody and GAPDH mouse monoclonal antibody (internal standard, Boster Ltd., Wuhan, China), followed by incubation with a horseradish peroxidase-conjugated secondary antibody (Kangcheng Bio-tech, China). Antibody binding was detected using peroxidase-conjugated secondary antibodies (Boster Ltd., Wuhan, China) for another $2 \mathrm{~h}$ at room temperature. The immunoreactive bands were visualized by western blot detection system ECL (Pierce Biotechnology, Rockford, IL, USA) and the intensity of the detected bands was analyzed using an Image J program.

2.8. Construction of Lentivirus and Infection. To knock down ANXA2 in AGS cells, a small hairpin RNA (shRNA) sequence, $5^{\prime}$-GGATGCTTTGAACATTGAA-3', was designed to target the ANXA2 gene and subcloned into the GV115 lentiviral vector (GeneChem Co., Ltd., Shanghai, China). The scrambled siRNA sequence $\left(5^{\prime}-\right.$ TTCTCCGAACGTGTCACGT- $3^{\prime}$ ) was used as the negative control. Subsequently, the lentiviral transfer vector plasmid, packaging plasmid, and envelope plasmid were transfected into human embryonic kidney (HEK) 293T cells (American Type Culture Collection, Manassas, VA, USA) using Lipofectamine 2000 reagent (Invitrogen Life Technologies, Carlsbad, CA, USA) for $48 \mathrm{~h}$. Then, cell supernatants containing lentivirus was harvested and concentrated. The virus titer was calibrated in the HEK293T cells. The lentivirus with a final concentration of $4 \times 10^{8} \mathrm{TU} / \mathrm{ml}$ was stored at $-80^{\circ} \mathrm{C}$. For lentivirus infection, according to the multiplicity of infection (MOI), cells grown to $30 \%$ confluence were transfected with lentivirus containing green fluorescent protein (GFP) reporter. The cell medium was changed to complete medium after $12 \mathrm{~h}$ of transfection. After cultured for another $3 \mathrm{~d}$, the infection rates were determined using fluorescence microscope.

2.9. Cell Proliferation Assay. Cell proliferation was tested by evaluating cell viability using the MTT assay. Briefly, cells seeded on 96 -well plates were stained with $20 \mu \mathrm{l} \mathrm{of} 5 \mathrm{mg} / \mathrm{ml}$ MTT dye (Beijing Dingguo Changsheng Biotechnology Co., Ltd., Beijing, China) per well for $4 \mathrm{~h}$ at $37^{\circ} \mathrm{C}$. After removal of the culture medium, $150 \mu \mathrm{l}$ of dimethyl sulphoxide (DMSO, Sinopharm Chemical Reagent Co., Ltd., Beijing, China) was 
added to solubilize the formazan. The absorbance values were determined at a wavelength of $495 \mathrm{~nm}$ in a BioTek ELx800 microplate reader. Three replicate wells were measured per assay, each experiment was performed at least 3 times.

2.10. Colony Formation Assay. Cells were plated on 6-well plates at an initial density of 800 cells per plate. The cells were cultured for up to $14 \mathrm{~d}$ or allowed to grow until most of colonies reached $>50$ cells per colony. After washing with phosphate buffered saline (PBS), the colonies were fixed with $10 \%$ paraformaldehyde for $30-60 \mathrm{~min}$, then stained with Giemsa and washed. Colony number was determined under an Olympus fluorescence microscopy equipped with MicroPublisher 3.3 RTV CCD camera.

2.11. Cell Apoptosis Assay. Flow cytometry was performed to detect cell apoptosis rates using Annexin V Apoptosis Detection Kit APC (eBioscience, San Diego, CA, USA) according to the manufacture's protocol. In brief, after washing the collected cells with PBS, the cells were incubated with $1 \times$ Annexin $\mathrm{V}$ binding buffer. Then $100 \mu \mathrm{l}$ cells $\left(1 \times 10^{5}-1 \times 10^{6}\right.$ cells) were stained with Annexin V-APC for $10 \mathrm{~min}$ at room temperature in the dark, we assessed the Annexin V-positive cells by a BD FACSCalibur flow cytometer.

2.12. Cell Cycle Analysis. Cells in culture were collected, resuspended in ice-cold PBS and fixed with $70 \%$ of icecold ethanol at $4^{\circ} \mathrm{C}$ for at least $1 \mathrm{~h}$. Then, the fixed wells were washed with PBS and stained with propidium iodide (PI, Sigma-Aldrich, St. Louis, MO, USA) and RNase A (Fermentas, St. Leon-Rot, Germany) in the dark at room temperature before analysiing the cell percentage in each cell cycle phase using a FACSCalibur instrument.

2.13. Matrigel Invasion Assay and Transwell Migration Assay. Cell invasion assay was tested using Biocoat Matrigel Invasion Chamber (Becton-Dickinson, Bedford, MA), a 24-well transwell unit with $8 \mu \mathrm{m}$ pore size polyethylene terephthalate membrane, according to the manufacturer's protocol. Briefly, $1 \times 10^{5}$ cells in the top chamber incubated in $100 \mu \mathrm{l}$ of fetal bovine serum- (FBS-) free medium were allowed to attach to 24-well transwell plates. The lower invasion chamber contained $600 \mu \mathrm{l}$ of RPMI-1640 medium with 30\% FBS. After $24 \mathrm{~h}$ of incubation, the cells that invaded the lower surface of the membrane were fixed and stained with Giemsa for 30 min. Under a phase-contrast microscope, we got the images of the cells in five predetermined fields at a magnification of 100 and 400. The stained cells were dissolved in $10 \%$ acetic acid $[16,17]$; the invasion was calculated by measuring the optical density (OD) values at a wavelength of $570 \mathrm{~nm}$ (OD 570).

Cells $\left(1 \times 10^{5}\right)$ incubated in $100 \mu \mathrm{l}$ of FBS-free medium were added to the 12-well format top chambers with 8.0 $\mu \mathrm{m}$ pore size porous transparent polyethylene terephthalate membrane. The lower chamber of the 12-well format transwell contained $600 \mu \mathrm{l}$ of RPMI-1640 medium with 30\% FBS. After incubation for $24 \mathrm{~h}$, the cells that invaded the lower surface of the membrane were stained with Giemsa. Pictures
TABLE 1: Positive correlated genes with ANXA2.

\begin{tabular}{lcc}
\hline Gene & $R$-value & $P$-value \\
\hline ANXA2P2 & 0.910 & $<0.005$ \\
S100A6 & 0.683 & $<0.005$ \\
S100A16 & 0.682 & $<0.005$ \\
S100A11 & 0.637 & $<0.005$ \\
GPRC5A & 0.627 & $<0.005$ \\
S100A10 & 0.615 & $<0.005$ \\
SFN & 0.602 & $<0.005$ \\
EZR & 0.598 & $<0.005$ \\
PLEK2 & 0.593 & $<0.005$ \\
EPHA2 & 0.583 & $<0.005$ \\
\hline
\end{tabular}

were taken under the microscope. The stained cells were dissolved in 10\% acetic acid and OD 570 was measured. The percentage of migration was evaluated by determining the OD570/MTT-OD490 ratio of the cells.

2.14. Wound Scratch Test. Cells in 96-well culture plates were grown in complete medium until cells reached 90-100\% confluence. A wound was scratched across the diameter of each plate. The cells were washed gently using PBS to remove cell debris. Cell migration was observed by microscopy at 0 , 8 , and $24 \mathrm{~h}$.

2.15. Statistical Analysis. Statistical comparisons between groups were determined by Student's t-test and chi-square test using SPSS 20.0 (SPSS Inc., Chicago, IL, USA). Statistical significance was assumed for $P<0.05$.

\section{Results}

3.1. Expression of ANXA2 in TCGA Database. By analyzing the expression level of ANXA2 in TCGA database, we found that ANXA2 was highly homogeneously expressed in GC tissues (Figure 1). The samples were divided into $\mathrm{T}$ stage (T1b, 14 samples; T2, 65 samples; T3, 181 samples, T4, 31 samples); N stage (N0, 123 samples; N1, 112 samples; N2, 80 samples; N3, 29 samples); and M stage (M0, 367 samples; M1, 27 samples). Then we analyzed the expression of ANXA2 in different stages. We found that the expression level of ANXA2 in patients with distant metastasis (M1) was higher than that in nondistant metastasis patients (M0). But there was no statistical significance in $\mathrm{T}$ stage and $\mathrm{N}$ stage (Figure 2).

3.2. Function of ANXA2 and Its Related Genes Analyzed by KEGG and GO Analysis. Using the R2 platform analysis, 415 samples were analyzed statistically; 3323 positive genes and 5700 negative genes were significantly correlated with ANXA2 expression. The top 10 positively and negatively correlated genes were shown in Tables 1 and 2. To determine the function of ANXA2 and its related genes, we used GO analysis and KEGG analysis (Tables 3 and 4). Biological processes included structure morphogenesis and movement of cells. Among the 9023 genes associated with ANXA2, 


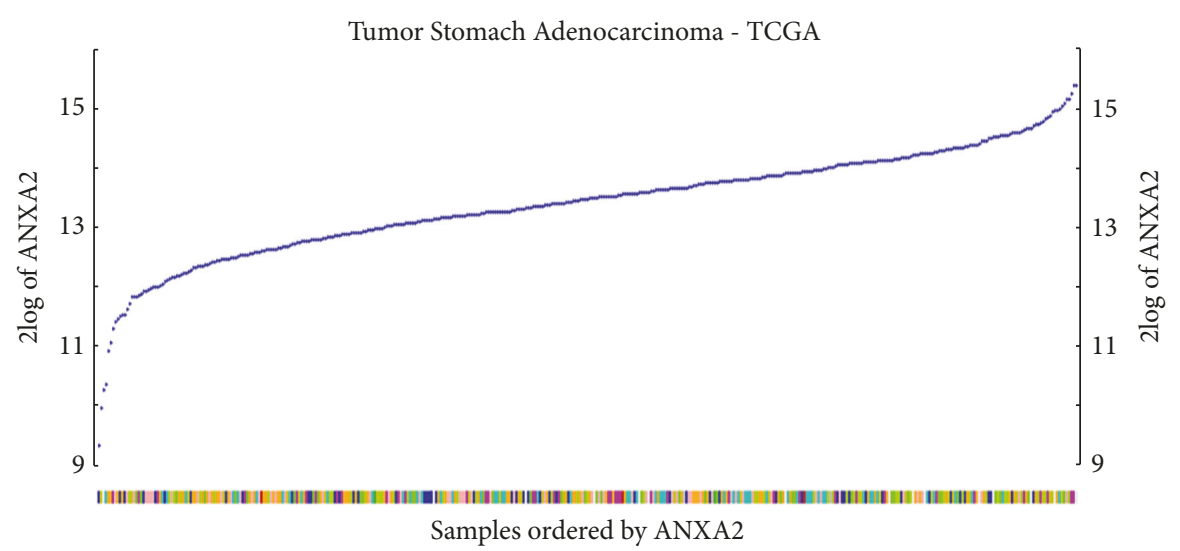

FIGURE 1: By analyzing the expression of ANXA2, we found ANXA2 was widely expressed in gastric cancer tissues. The blue points showed the expression level ANXA2. Different colors of X-Line indicated gastric cancer patients with different stages.
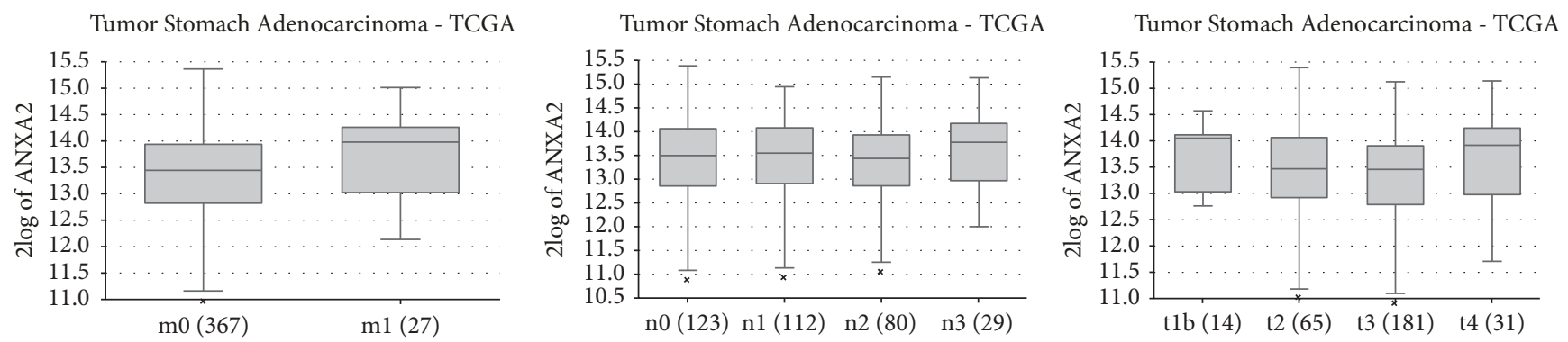

FIgURE 2: Differential expression of ANXA2 could differentiate $\mathrm{M}$ staging of gastric cancer patients $(P=0.04)$, not $\mathrm{T}$ staging or $\mathrm{N}$ staging.

TABLE 2: Negative correlated genes with ANXA2.

\begin{tabular}{lcc}
\hline Gene & $R$-value & $P$-value \\
\hline CBFA2T2 & -0.561 & $<0.005$ \\
CHD6 & -0.559 & $<0.005$ \\
ZFHX3 & -0.540 & $<0.005$ \\
BSN & -0.529 & $<0.005$ \\
NTN3 & -0.513 & $<0.005$ \\
ZNF445 & -0.508 & $<0.005$ \\
IQSEC1 & -0.505 & $<0.005$ \\
CBX6 & -0.486 & $<0.005$ \\
SEZ6 & -0.481 & $<0.005$ \\
IRF2BPL & -0.480 & $<0.005$ \\
\hline
\end{tabular}

KEGG analysis found that 3006 genes were involved in 47 signaling pathways; most of them were related to pathways in cancer and regulation of actin cytoskeleton $(P<0.005)$.

Among the genes coexpressed with ANXA2, we found that the signaling pathways 'Focal adhesion' and 'Regulation of actin cytoskeleton' were activated, which was the same as we anticipated (Figure 3). This actin polymerization may change cytoskeleton. On the other hand, the filamentous filopodia may help the movement of tumor cells.

3.3. Expression of ANXA2 in GC Tissues and Adjacent Tissues. Our experiments summarized the different expression level of ANXA2 in GC tissues and adjacent tissues based on immunohistochemical analysis. ANXA2 mainly expressed in GC tissues with obvious brownish yellow particles (Figure 4(d)). Negative ANXA2 expression was detected in 29 (32.2\%) of the 90 GC tissues samples, 6 samples (6.7\%) were hadropositive and 25 samples (27.8\%) were positive ANXA2 expression (Table 5(a)). The remaining samples were weakly positive. In contrast to GC tissues, $91.1 \%$ of the adjacent tissues showed negative ANXA2 expression (Figure 4(c)). The data showed that the distribution of ANXA2 expression in GC and adjacent tissues was statistically significant $(P<0.001$, Table 5(b)).

3.4. Expression of ANXA2 in Different GC Cell Lines. Expression level of ANXA2 mRNA in human GC cell lines with different grade of differentiation was examined by quantitative real-time PCR. The results showed that ANXA2 mRNA was positively expressed in four human GC cells (SGC-7901, BGC-823, MKN-45, and AGS cells), the highest expression of ANXA2 mRNA was tested in AGS cells (Figure 5(a)). Consistent with mRNA expression level, AGS cell had the highest expression level of ANXA2 protein detected by western blot (Figure 5(b)).

3.5. Expression of ANXA2 $\mathrm{mRNA}$ and Protein in AGS Cells. To suppress ANXA2 expression, AGS cells were infected with a lentivirus which expressed ANXA2-spcific siRNA and GFP. 
TABLE 3: GO analysis of ANXA2 in the database.

\begin{tabular}{lccc}
\hline Biological process & No. of genes & $P$-value & Go path no. \\
\hline membrane-bounded organelle & 5877 & $<0.005$ & 43227 \\
anatomical structure morphogenesis & 1332 & $<0.005$ & $<653$ \\
cell morphogenesis & 553 & $<0.005$ & 902 \\
protein binding & 5369 & $<0.005$ & 5515 \\
movement of cell or subcellular component & 1006 & $<0.005$ & 6928 \\
cytoskeleton organization & 673 & $<0.005$ & 7010 \\
\hline
\end{tabular}

TABLE 4: KEGG analysis of ANXA2 in the database.

\begin{tabular}{|c|c|c|c|}
\hline Biological process & No. of genes & $P$-value & Related genes \\
\hline Pathways in cancer & 224 & $<0.005$ & $\begin{array}{l}\text { APPL1, FZD10, LPAR6, GNA13, CXCL12, ABL1, } \\
\text { PIK3R5, AKT3, MTOR, STAT5A, ARAF, BCL2, } \\
\text { SMAD4, TGFBR1, MSH3, PIAS2... }\end{array}$ \\
\hline Focal adhesion & 122 & $<0.005$ & $\begin{array}{c}\text { EGF, EGFR, PIK3R5, ITGA11, LAMC3, PAK4, DOCK1, } \\
\text { ROCK1, GRF2, MAPK8, JUN, BLC2, ELK1, BRAF, } \\
\text { RAPGEF1, CRK, SOS1... }\end{array}$ \\
\hline HIF 1 signaling pathway & 67 & $<0.005$ & $\begin{array}{l}\text { AKT2, AKT3, ANGPT1, ARNT, BCL2, CAMK2A, } \\
\text { CAMK2B, EGLN1, ENO3, EP300, EPO, PIK3CD, } \\
\text { PIK3CG, TEK, TF, VHL... }\end{array}$ \\
\hline Proteoglycans in cancer & 116 & $<0.005$ & $\begin{array}{l}\text { ELK1, ROCK1, TIAM1, ITPR1, DROSHA, HOXD10, } \\
\text { PIK3R5, AKT3, PDPK1, MTOR, PDCD4, COL21A1, } \\
\text { ARAF, HGF, FRS2, SOS1, WNT16... }\end{array}$ \\
\hline Regulation of actin cytoskeleton & 118 & $<0.005$ & $\begin{array}{c}\text { F2, GNA13, ARAF, PIK3R5, SOS1, EGFR, EGF, ITGA11, } \\
\text { CHRM1, FGD1, VAV3, MSN, ACTN4, MYLK, MYH9, } \\
\text { CFL1, ENAH, WASF1... }\end{array}$ \\
\hline Central carbon metabolism in cancer & 42 & $<0.005$ & $\begin{array}{c}\text { AKT2, FGFR1, FLT3, GCK, KIT, MTOR, NRAS, } \\
\text { NTRK1, NTRK3, PDGFRA, PFKM, PGAM2, PIK3CD, } \\
\text { RET... }\end{array}$ \\
\hline Vascular smooth muscle contraction & 76 & $<0.005$ & $\begin{array}{c}\text { ACTA2, ADRA1A, AGTR1, BRAF, CACNA1C, } \\
\text { EDNRA, GNA12, GNAS, ITPR1, KCNMA1, MRVI1, } \\
\text { PLA2G12A, PLCB4, RAMP3, ROCK1... }\end{array}$ \\
\hline
\end{tabular}

* For the "Biological process" column, we showed the function of ANXA2 in gastric cancer tissue. The "No. of genes" column showed the number of genes that enriched in corresponding pathways, and the details were in the "Related genes" column. Correlation statistics were calculated by the R2 platform.

TABLE 5: The expression of ANXA2 in GC tissues.

(a)

\begin{tabular}{|c|c|c|c|c|c|}
\hline \multirow[b]{2}{*}{ Tissue type } & \multirow[b]{2}{*}{ Number of cases } & \multicolumn{4}{|c|}{ ANXA2 expression } \\
\hline & & $\begin{array}{c}\text { Negative } \\
(-)\end{array}$ & $\begin{array}{c}\text { Weakly positive } \\
(+)\end{array}$ & $\begin{array}{c}\text { Positive } \\
(++)\end{array}$ & $\begin{array}{l}\text { Hadro-positive } \\
\qquad(+++)\end{array}$ \\
\hline Adjacent & 90 & $\begin{array}{c}82 \\
(91.1 \%)\end{array}$ & $\begin{array}{c}4 \\
(4.4 \%)\end{array}$ & $\begin{array}{c}3 \\
(3.3 \%)\end{array}$ & $\begin{array}{c}1 \\
(1.1 \%)\end{array}$ \\
\hline Cancer & 90 & $\begin{array}{c}\downarrow 29 \\
(32.2 \%)\end{array}$ & $\begin{array}{c}\uparrow 20 \\
(22.2 \%)\end{array}$ & $\begin{array}{c}\uparrow 25 \\
(27.8 \%)\end{array}$ & $\begin{array}{c}\uparrow 6 \\
(6.7 \%)\end{array}$ \\
\hline
\end{tabular}

* $\downarrow$ Decreased ANXA2 expression in gastric cancer tissue compared to adjacent tissue.

$\uparrow$ Enhanced ANXA2 expression in gastric cancer tissue compared to adjacent tissue.

(b)

\begin{tabular}{lcccc}
\hline Cancer & Total Number & Adjacent & & \\
& & Negative & Total positive & \\
\hline Negative & 29 & 28 & 1 & $<$ \\
Positive & 61 & 54 & 7 & $<0.001$ \\
\hline
\end{tabular}




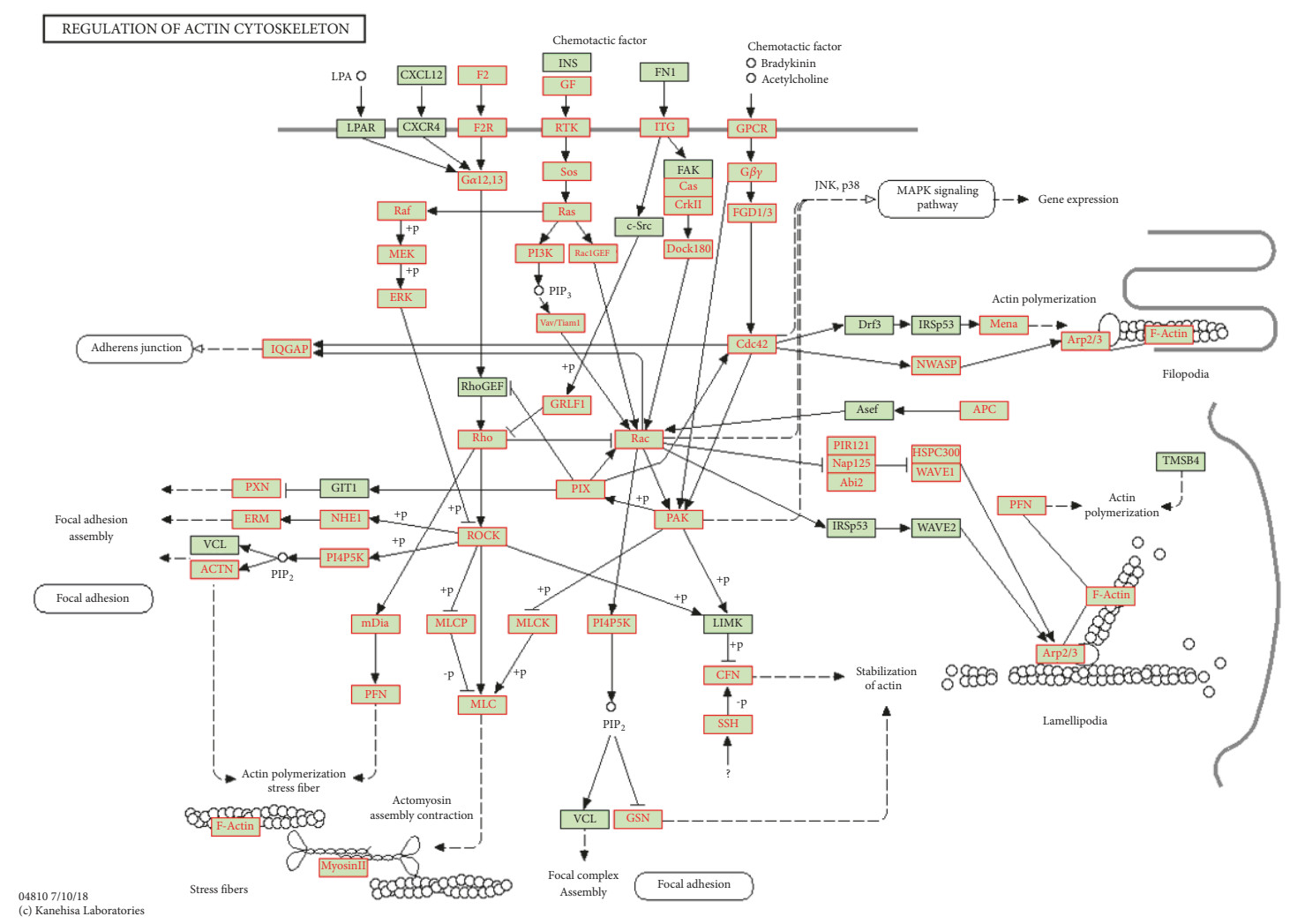

(a)

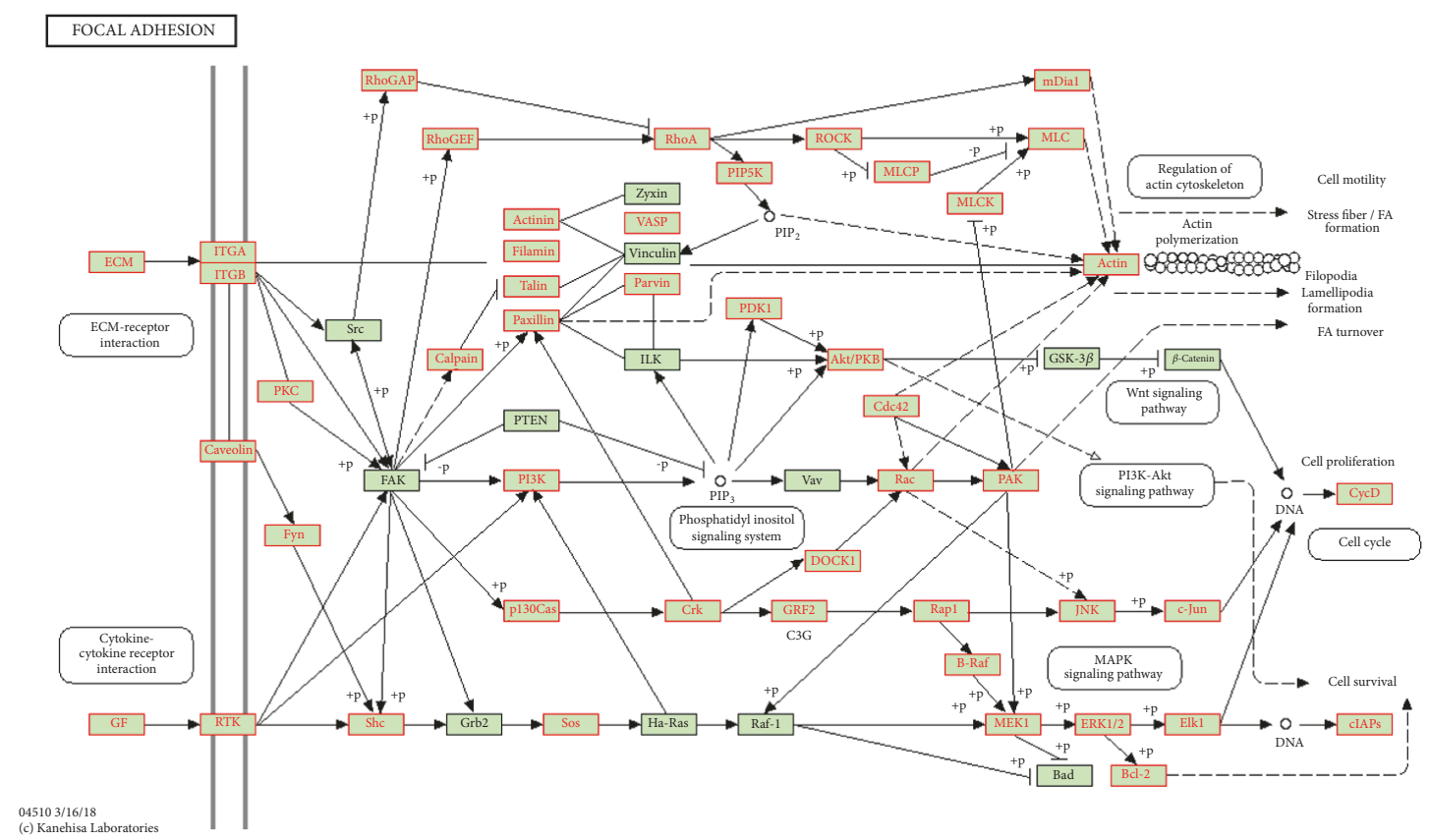

(b)

Figure 3: (a) By KEGG pathway enrichment analysis, ANXA2 upregulated filopodia pathway, resulting in cell metastasis. Microfilament is one component of cytoskeleton; it is mainly composed of actin. ANXA2 upregulated regulation of actin cytoskeleton pathway and led to the rise of cell deformability. (b) By KEGG pathway enrichment analysis, ANXA2 upregulated focal adhesion pathway, resulting in cell motility, proliferation and survival. 


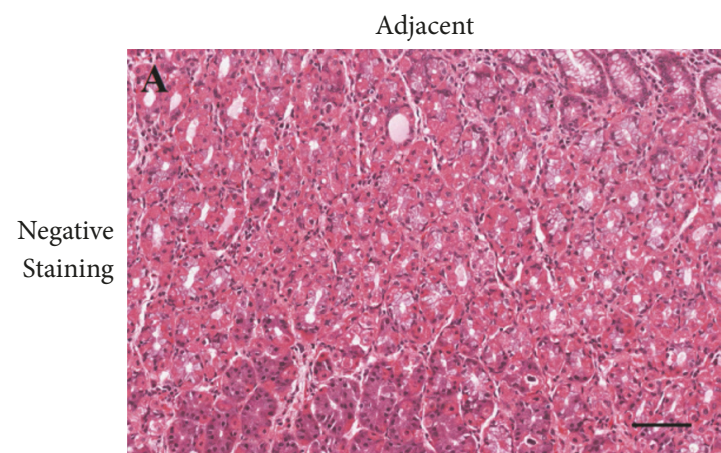

(a)

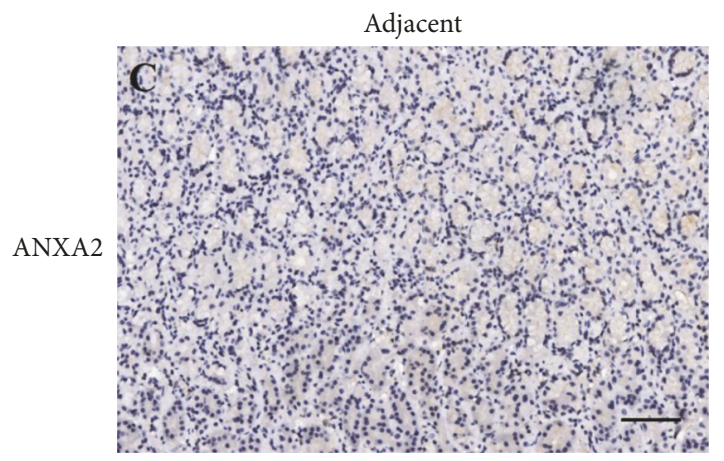

(c)

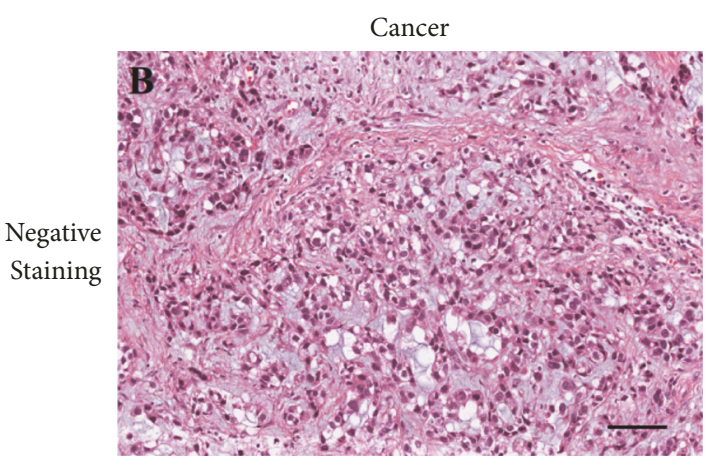

(b)

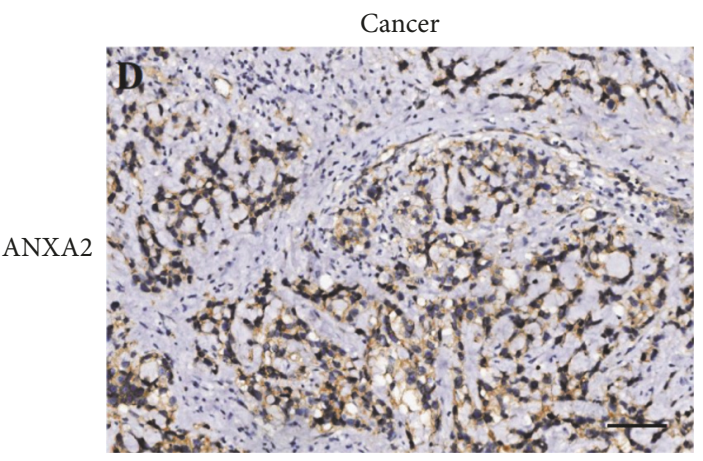

(d)

FIGURE 4: Immunohistochemical staining of ANXA2 was upregulated in gastric cancer tissue than adjacent tissue. Negative staining of (a) adjacent tissue and (b) gastric cancer tissue. ANXA2 expression of (c) adjacent tissue and (d) gastric cancer tissue (magnification, 100×, scale bar, $50 \mu \mathrm{m})$.

After $72 \mathrm{~h}$ infection, more than $80 \%$ of cells expressed GFP, indicating successful infection (Figure 5(c)). ANXA2 mRNA (Figure 5(d)) and ANXA2 protein expression (Figure 5(e)) in AGS cells infected with lentivirus expressing ANXA2 siRNA (knock-down group) were significantly lower than in cells infected with lentivirus expressing scrambled siRNA (negative control group) and AGS cells (control group). These results demonstrated that siRNA directed towards ANXA2 was efficient in specifically knocking down the ANXA2 gene in AGS cells.

3.6. The Function of ANXA2 on Proliferation in AGS Cells. As illustrated in Figures 6(a) and 6(b), compared with control and negative control groups, numbers of cells colony formation capacity significantly reduced in ANXA2 knock-down group. The MTT assay showed obviously less proliferation in ANXA2 knock-down group at 4 and $5 \mathrm{~d}$ after infection than other groups (Figure 6(c)). These findings totally revealed that ANXA2 was essential for AGS cell proliferation. ANXA2 silencing caused G1 phase arrest of AGS cells and a decline in the number of cells in the $S$ and $\mathrm{G} 2 / \mathrm{m}$ phases compared to negative control and control cells (Figure 6(d)). The rates of apoptosis in ANXA2-silencing cells were appreciably increased (Figure 6(e)).

3.7. The Function of ANXA2 on Invasion and Migration in AGS Cells. Matrigel invasion chamber, transwell, and scratch assay provided evidence that ANXA2 silencing inhibited invasion and migration ability in ANXA2 knock-down group than in control and negative control groups (Figures 7(a) and $7(\mathrm{~b})$ ). The results showed that the migration rate of ANXA2 knock-down group cells was significantly decreased at $24 \mathrm{~h}$ than in negative control and control cells (Figures $7(\mathrm{c})$ and $7(\mathrm{~d}))$.

\section{Discussion}

GC is a heterogeneous disease with a high mortality rate. Its poor prognosis is mainly contributed by extensive invasion and metastasis [18]. The mechanism of tumor metastasis is complex. It includes cancerous cells away from their primary tumor, the intravasation into the bloodstream or lymph nodes, the transit through the tumor microenvironment, and the aggregation to secondary tissues. Cancer cell migration plays an important role in the metastatic process. However, the specific reasons have not been clarified yet. Therefore, indepth research on genes related to invasion and metastasis of GC is crucial.

ANXA2 is overexpressed in most tumor tissues and acts as a pivotal part in tumor development. Prior research generally confirms that the expression level of ANXA2 has a close relation with progression of tumors [19-21]. Zhang et al. [14] prove that expression level of ANXA2 mRNA is higher in GC tissues than nontumor tissues. Besides, 


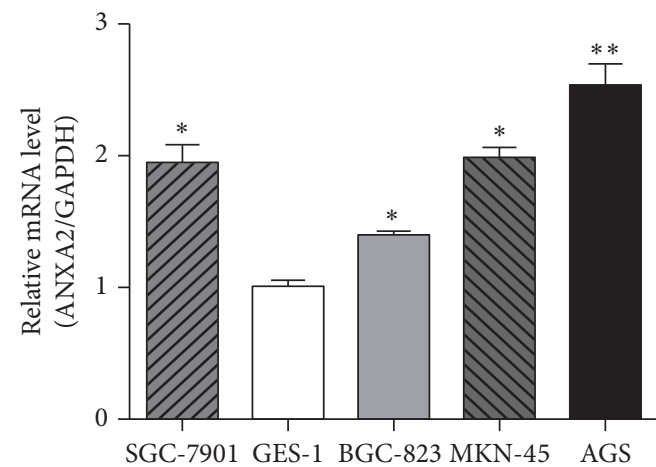

(a)
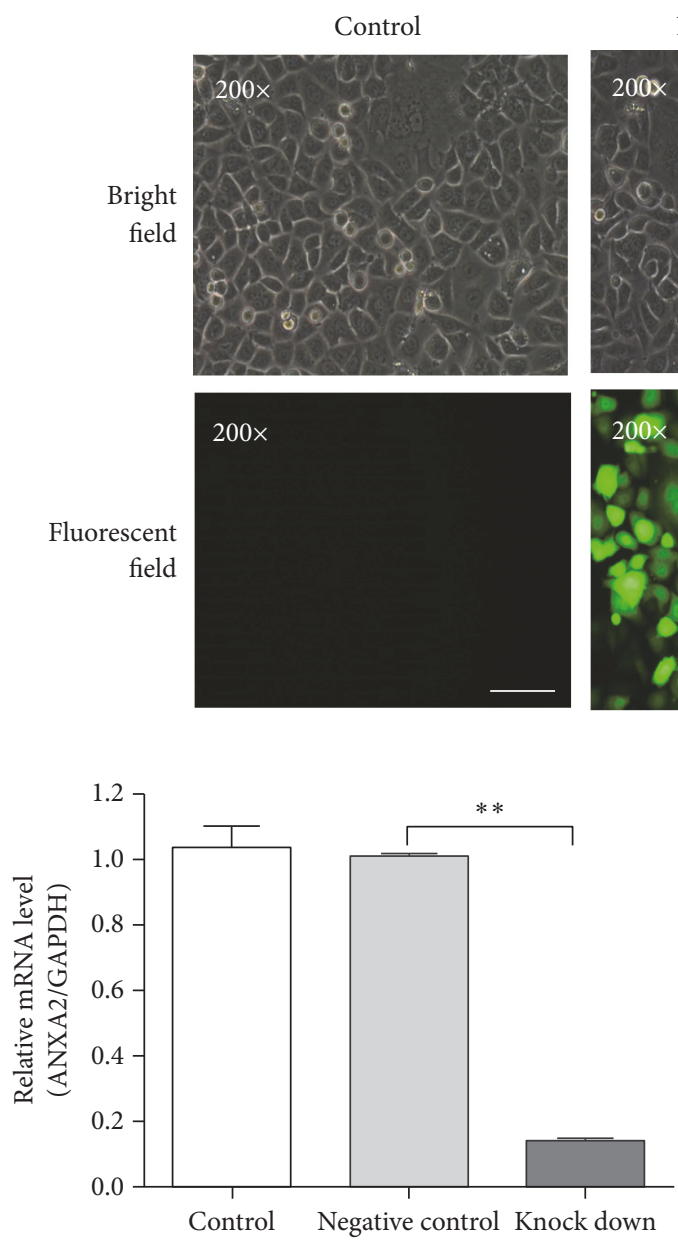

(d)
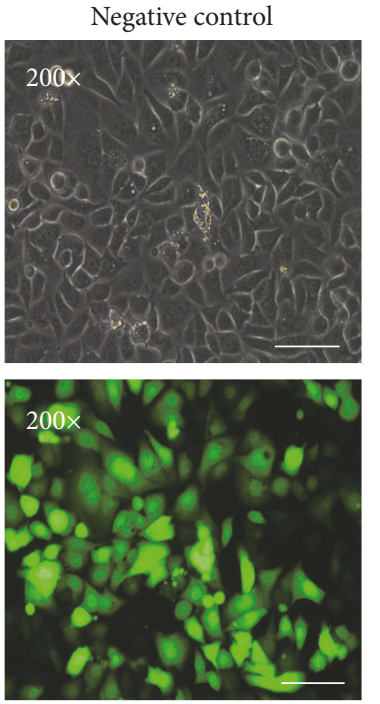

(c)
SGC-7901 GES-1 BGC-823 MKN-45 AGS

ANXA2

GAPDH

(b)

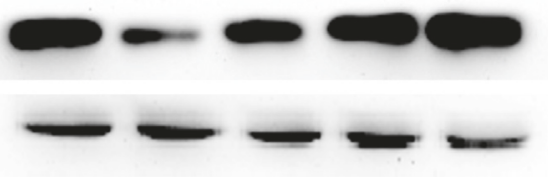

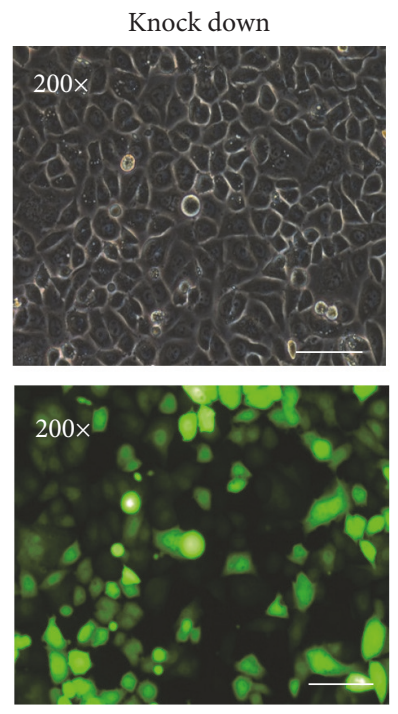

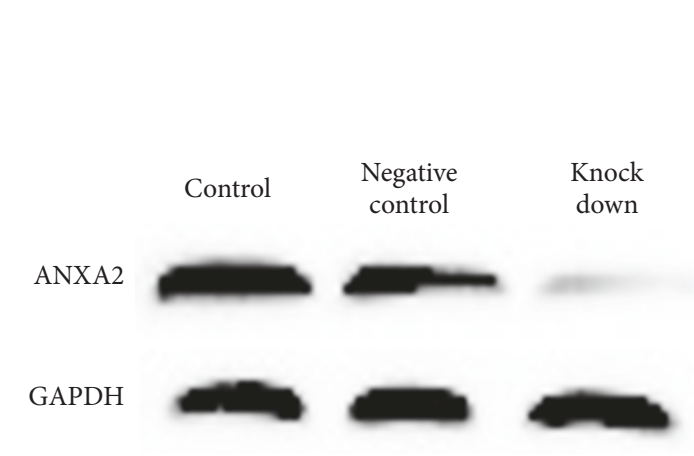

(e)

FIGURE 5: ANXA2 siRNA suppressed the ANXA2 mRNA and protein expression levels in gastric cancer cell lines. (a) Quantitative realtime PCR analyzed ANXA2 mRNA levels in different gastric cancer cell lines including SGC-7901, GES-1, BGC-823, MKN-45, and AGS. $* P<0.05, * * P<0.01$ compared to GES-1. (b) The expression level of ANXA2 protein in SGC-7901, GES-1, BGC-823, MKN-45, and AGS cells was determined using western blot. GAPDH was utilized as the internal control. (c) Bright and GFP fluorescent field of AGS cell $72 \mathrm{~h}$ after infection with negative control (middle panel) or lentivirus containing ANXA2-RNAi (knock-down, right panel), magnification, 200×, scale bar, $100 \mu \mathrm{m}$. (d) Quantitative real-time PCR assessment of ANXA2 mRNA levels in AGS siRNA-infected cells compared to control and negative control cells, $* * P<0.01$. (e) Western blot analysis of ANXA2 protein expression in ANXA2 knock-down cells compared to control and negative control cells. 


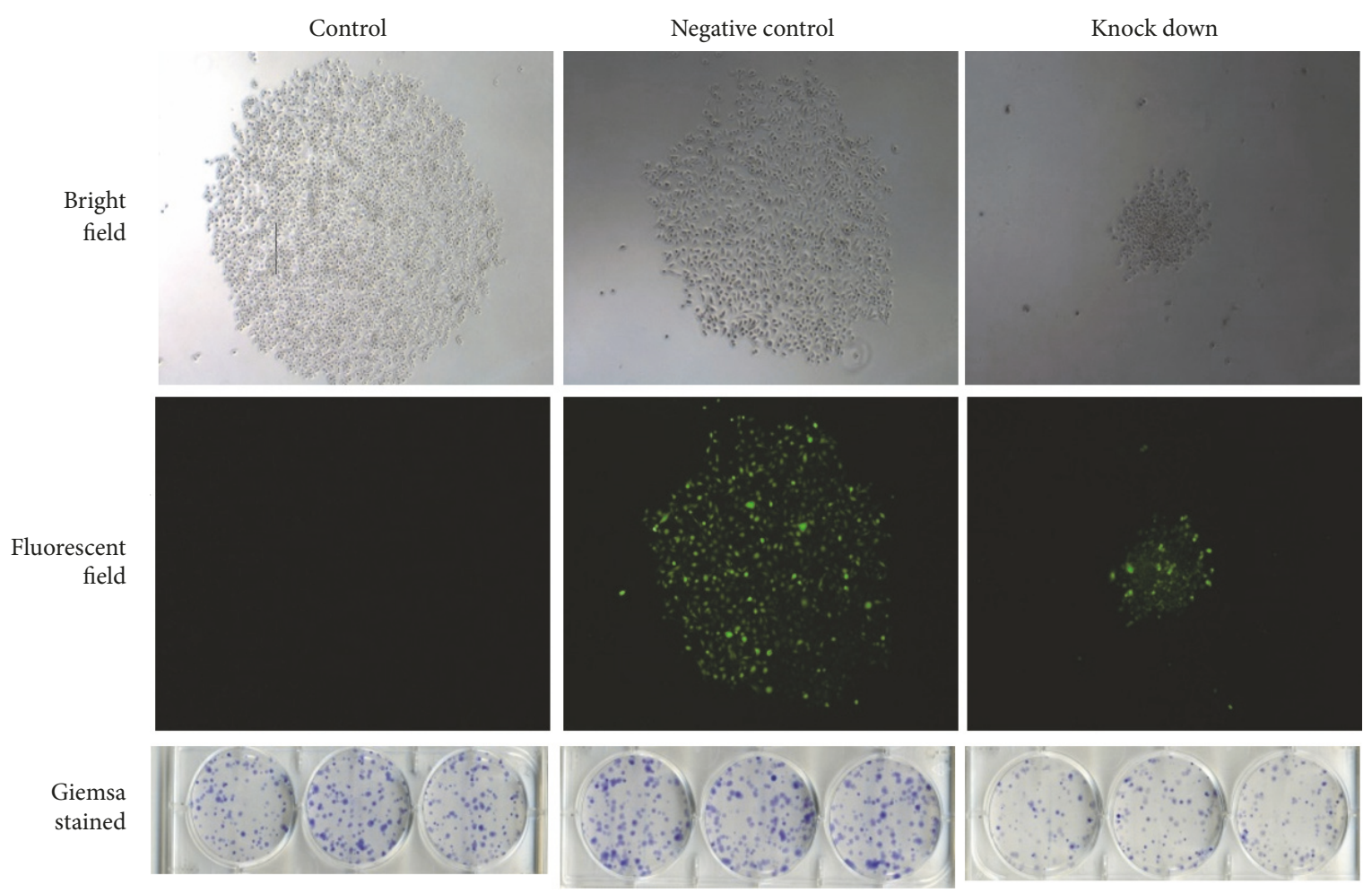

(a)

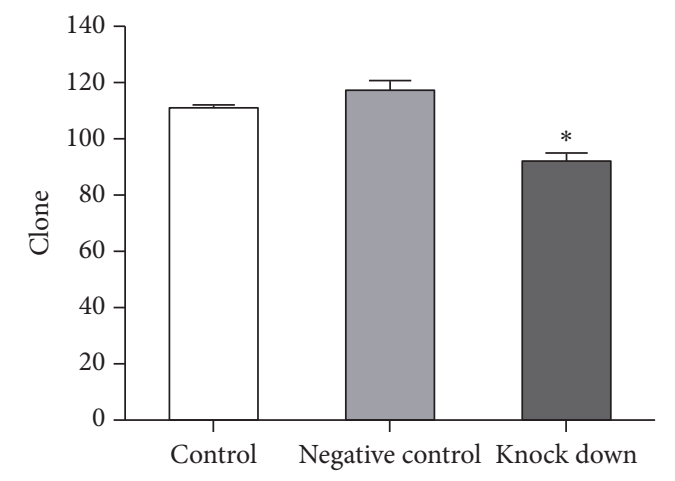

(b)

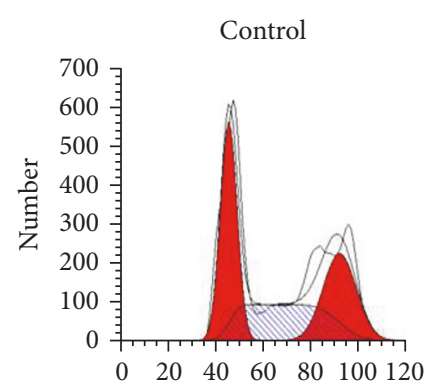

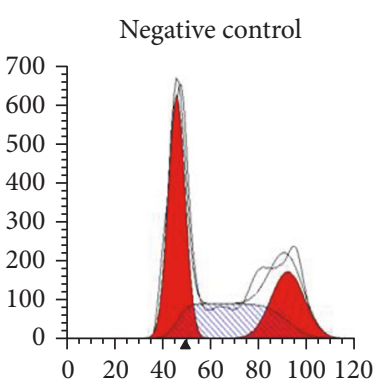

(d)

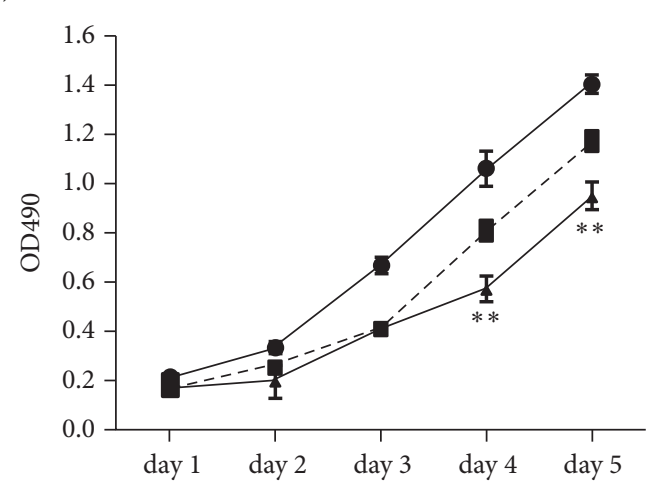

- - Control

- - Negative control

$\rightarrow$ Knock down

(c)
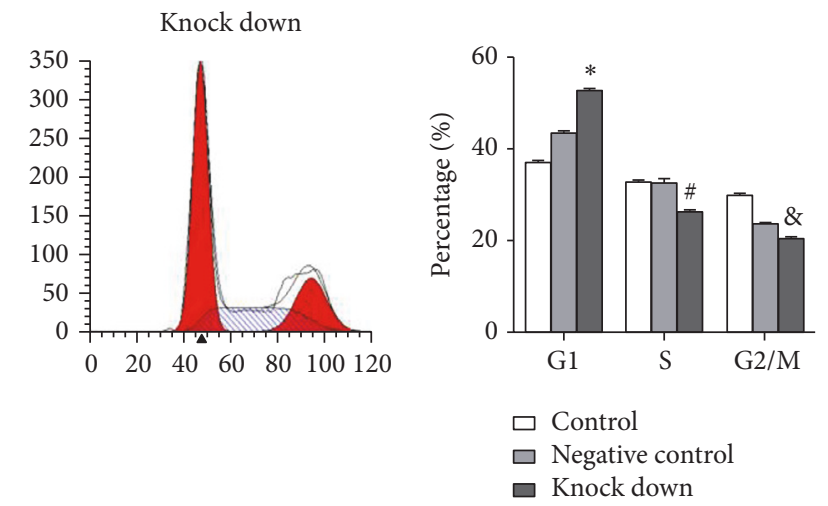

FIgure 6: Continued. 

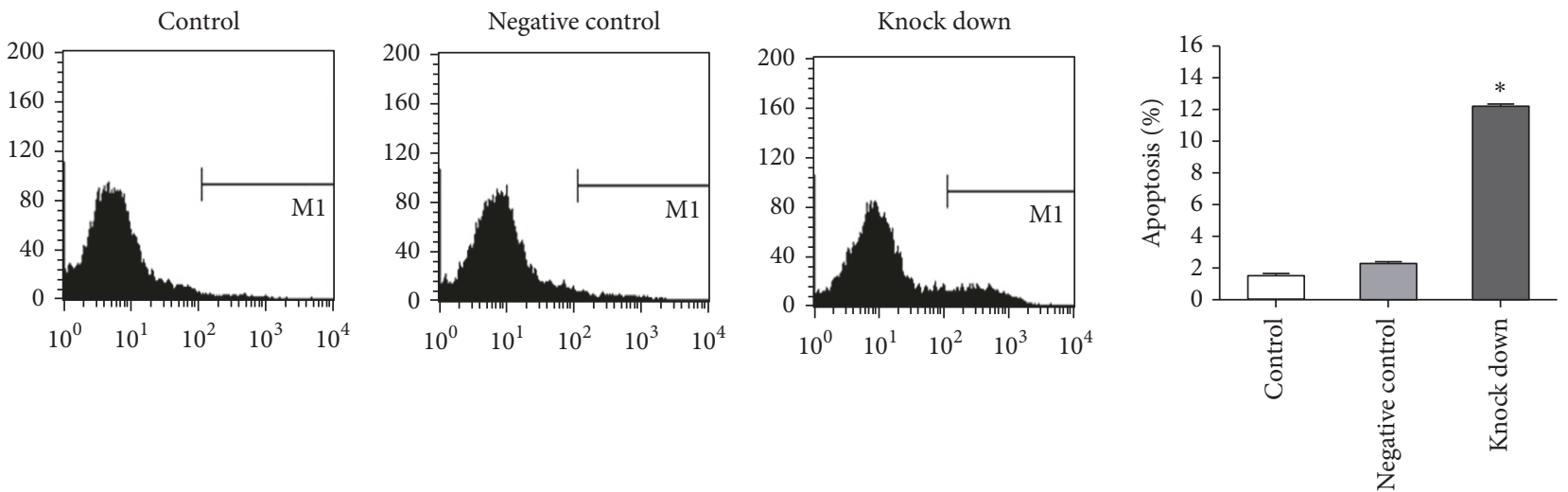

(e)

FIGURE 6: ANXA2 silencing inhibited AGS cell growth. (a) Representative size of cell colonies, bright field (upper panel), fluorescent field (middle panel), and Giemsa stained (lower panel), $P<0.05$. (b) The number of cell colonies in control, negative control, and ANXA2 knockdown cells, $* P<0.05$. (c) Using MTT assay, the relative AGS cell proliferation pattern at different time points was investigated, $* P<0.05, \mathrm{n}=5$. (d) The ratio of cells at different cell cycle phases in control, negative control, and ANXA2 knock-down groups. (e) The cell apoptotic rate in control, negative control, and ANXA2 knock-down groups using flow cytometry, $* P<0.05, \mathrm{n}=3$.

as the expression level of ANXA2 increases, the degree of pathological differentiation of GC cells decreases [22]. Consistent with previous study, our research demonstrated that ANXA2 was significantly enhanced in GC tissues compared with adjacent tissues.

Previous reports have shown the effect of ANXA2 in many cancerous cells. For example, ANXA2 may promote the progression and invasion of human lung cancer cell [23]. With the upregulation of ANXA2, the potential ability of metastatic and invasive is increased in hepatoma cell, shRNA-mediated ANXA2 silencing significantly inhibits cell invasion, migration, and tumorigenic potential [24]. Similar studies have studied ovarian cancer cell [25], glioma cell [12], and breast cancer cell [26]. However, there is no relevant study on biological behavior of ANXA2 in GC cells.

We first analyzed the expression of ANXA2 in GC tissue from TCGA database; it made a new view for the research of GC patients. Theoretically, the large size of the RNASeq dataset may improve the reliability of the results. Thus, data analysis depending on RNA-Seq can provide a more realistic context for us. It was interesting that we first verified our conjecture from TCGA database. The high expression of ANXA2 activated tumor cells to generate cytoskeleton, acted a positive role in proliferation of GC cells. On the other hand, the high expression of ANXA2 activated the pathway for tumor cells to grow filopodia, it provided energy for cancerous cells to move themselves. From this perspective, we explained why the high expression of ANXA2 indicated poor prognosis of GC.

In our study, AGS cell line displayed the highest expression level of ANXA2 among the four kinds of human GC cell lines and it was selected as a cell model for subsequent experiments. To elucidate roles of ANXA2 in GC cell growth, AGS cells were infected with a lentivirus expression ANXA2spcific siRNA and GFP, it laid the foundation for the further experiments.
Our results observed that the proliferation and colony formation ability of lentivirus-mediated Annexin A2 knockdown cells were suppressed appreciably; it prompted us to consider that ANXA2 might act as a positive role in proliferation of GC cells in vitro. ANXA2 knock-down cells showed a significant more proportion of cells in the G1 phase and a decline number in the $S$ and $\mathrm{G} 2 / \mathrm{m}$ phases compared to negative control or control cells. Similarly, in previous study, non-small cell lung cancer cell lines with ANXA2 silencing showed inhibition both in tumor growth and cell proliferation and also induced cell cycle arrest at the G2 phase [27]. Apoptosis rates apparently increased in ANXA2 knockdown cells. Abnormal acceleration of cell cycle and inhibition of apoptosis are important causes of the tumor cell growth. These results suggested that ANXA2 was a promising target for inhibiting GC cell proliferation possible by blocking the cell cycle and promoting cell apoptosis.

Invasion and metastasis as the important biological behaviors of malignant tumor are the leading causes for poor prognosis of GC patients [28]. Numerous factors contributed to regulate invasion and metastasis and other biological behaviors in tumor cells. Our experiment showed that knocking down ANXA2 expression inhibited invasion and migration ability of AGS cells to achieve the purpose of delaying the progression of GC.

In our previous published study, we used gene expression microarray and detected 100 differentially expressed genes including 58 upregulated and 42 downregulated human genes. According to all pathway genetic information in KEGG and BIOCARTA, we did the enrichment analysis of the difference of gene; the important ANXA2 related genes including FGA, FGB, SERPINB2, CD55, PLAUR, MET, RAP1A, and ETS1 were screened. Western blot showed that after silencing of ANXA2, RAP1A, and MAP2K1 protein expression decreased in AGS cells. We speculated that RAP1A and MAP2K1 might be potential downstream regulating 

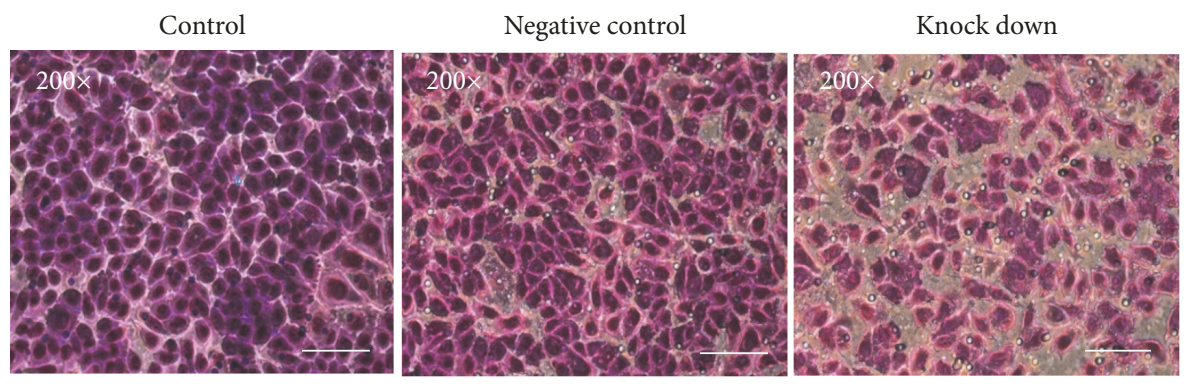

(a)
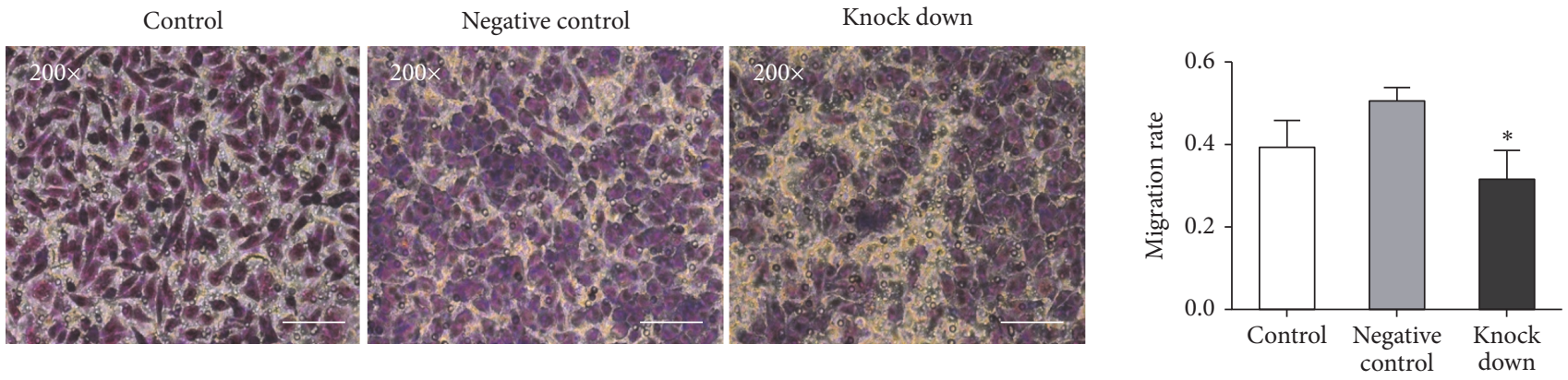

(b)
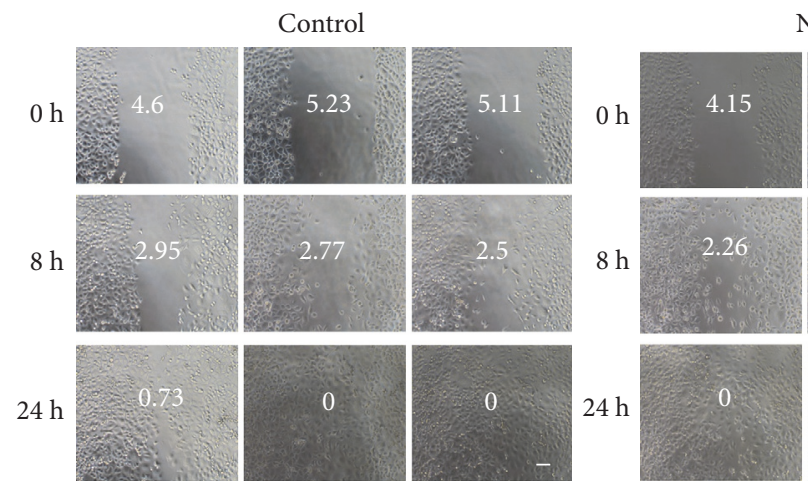

Negative control
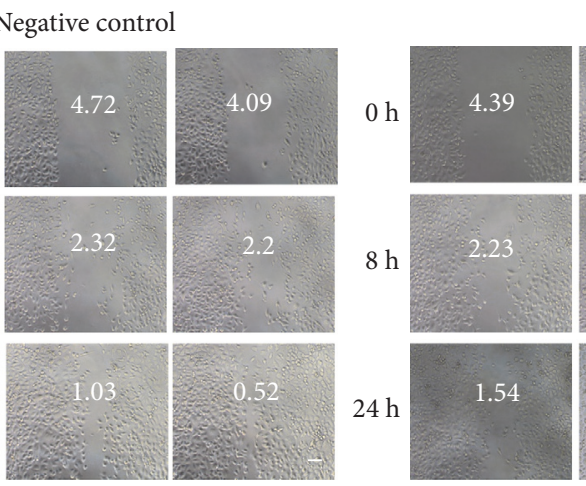

Knock down
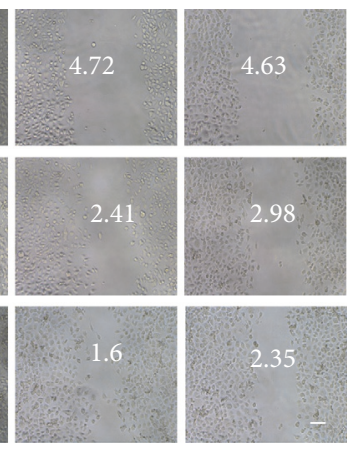

(c)

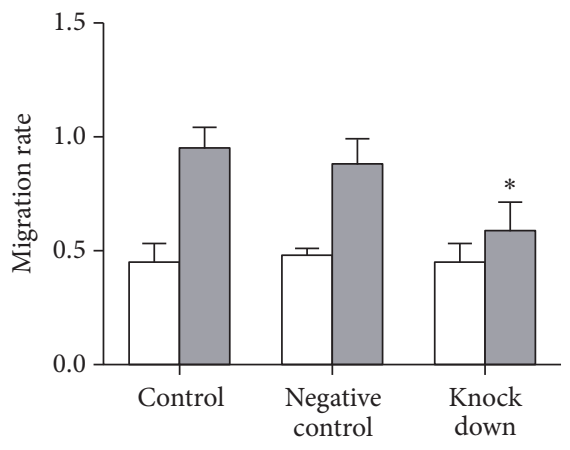

$\square 8 \mathrm{~h}$

(d)

FIGURE 7: ANXA2 silencing inhibited invasion and migration rate of AGS cells. (a) Giemsa staining and compared with negative control group, the invasion rate in knock-down group was decreased, $* P<0.05, \mathrm{n}=3$, scale bar, $100 \mu \mathrm{m}$. (b) Giemsa staining and compared with negative control group, the migration rate in knock-down group was decreased, $* P<0.05, \mathrm{n}=3$, scale bar, $100 \mu \mathrm{m}$. (c) Picture of cell scratch, scale bar, $100 \mu \mathrm{m}$. (d) In $8 \mathrm{~h}$, the migration rate has no significant difference in control, negative control, and knock-down groups. Compared with control group and negative control group, at $24 \mathrm{~h}$, the migration rate of knock-down group was lower, $* P<0.05, \mathrm{n}=3$. 
genes of ANXA2 taking part in MAPK signaling pathway in AGS cells [29].

Identifying target points of genes related to proliferation, invasion, and migration of GC is essential to consummate traditional treatments and improve prognosis of patient. Our experimental results provided further insights into the research progression on biological behavior of GC. It could lead researchers to taking serious consideration of the potential benefit of ANXA2 silence as an effective antitumor therapeutic tool.

\section{Conclusions}

In conclusion, our results presented a compelling evidence for the role of ANXA2 in biological behavior of GC. Analyzing the TCGA datasets, we found that ANXA2 promoted cell migration and proliferation in transcriptome level. The ANXA2 protein and mRNA expression in GC tissues were significantly upregulated. Lentivirus-mediated ANXA2 silencing affected the biological behavior of AGS cells through inhibiting the proliferation, invasion, and migration, promoting apoptosis, and arresting cell cycle.

\section{Abbreviations}

ANXA2: Annexin A2

GC: $\quad$ Gastric cancer

TCGA: The Cancer Genome Atlas

GO: $\quad$ Gene Ontology

KEGG: Kyoto Encyclopedia of Genes and Genomes

q-PCR: Quantitative PCR

PBS: $\quad$ Phosphate buffered saline

OD: Optical density.

\section{Data Availability}

Figures 1, 2, and 3 data used to support the findings of this study have been deposited in the TCGA repository.

\section{Conflicts of Interest}

The authors declare that there are no conflicts of interest associated with the manuscript.

\section{Authors' Contributions}

Rui Xie and Jia Liu contributed equally to this work. Rui Xie, Jiuxin Zhu, and Tianyi Fang designed and conceived this project; Tianyi Fang performed bioinformatics analysis. Jia Liu, Yufeng Wang, Lijuan Wang, and Wei Yang performed the experiments; Jiahe $\mathrm{Hu}$, Xinyan Huang, Peiqiang Liang, and Hong Sui contributed in the materials finding and purchasing and reagents preparation. Ping Liu, Xuefeng Yu, and Chunfeng Li analyzed the data (including statistical analysis); Rui Xie, Jia Liu, Yuxian Bai, and Yingwei Xue drafted and revised the manuscript. All authors approved the final version for publication and agreed to be accountable for all aspects of the work in ensuring that questions related to the accuracy or integrity of any part of the work are appropriately investigated and resolved.

\section{Acknowledgments}

This work was supported by the Yu Weihan Academician's Outstanding Youth Training Fund in Harbin Medical University, Nn10 program (Nn10 PY 2017-03), Youth Elite Training Fund (JY2015-02), Outstanding Youth Fund (JCQN 201804) in Harbin Medical University Cancer Hospital, and Natural Science Foundation of Heilongjiang Province of China (QC2013C077).

\section{References}

[1] J. Ferlay, I. Soerjomataram, R. Dikshit et al., "Cancer incidence and mortality worldwide: sources, methods and major patterns in GLOBOCAN 2012," International Journal of Cancer, vol. 136, no. 5, pp. E359-E386, 2014.

[2] H. J. Meyer and H. Wilke, "Treatment strategies in gastric cancer," Deutsches Aerzteblatt Online, vol. 108, no. 41, pp. 698705, 2011.

[3] S. C. Park and H. J. Chun, "Chemotherapy for advanced gastric cancer: Review and update of current practices," Gut and Liver, vol. 7, no. 4, pp. 385-393, 2013.

[4] Y. J. Bang, Y. W. Kim, H. K. Yang et al., "Adjuvant capecitabine and oxaliplatin for gastric cancer after D2 gastrectomy (CLASSIC): a phase 3 open-label, randomised controlled trial," Lancet, vol. 379, no. 9813, pp. 315-321, 2012.

[5] K.-W. Lee, S. R. Park, D.-Y. Oh et al., "Phase I study of sunitinib plus capecitabine/cisplatin or capecitabine/oxaliplatin in advanced gastric cancer," Investigational New Drugs, vol. 31, no. 6, pp. 1547-1558, 2013.

[6] A. Stein, D. Arnold, P. C. Thuss-Patience et al., "Docetaxel, oxaliplatin and capecitabine (TEX regimen) in patients with metastatic gastric or gastro-esophageal cancer: Results of a multicenter phase I/II study," Acta Oncologica, vol. 53, no. 3, pp. 392-398, 2014.

[7] T. P. Batista, C. A. A. L. de Santos, and G. F. G. Almeida, "Perioperative chemotherapy in locally advanced gastric cancer," Arquivos de Gastroenterologia, vol. 50, no. 3, pp. 236-242, 2013.

[8] N. A. Lokman, M. P. Ween, M. K. Oehler, and C. Ricciardelli, "The role of annexin A2 in tumorigenesis and cancer progression," Cancer Microenvironment, vol. 4, no. 2, pp. 199-208, 2011.

[9] H.-J. Zhang, D.-F. Yao, M. Yao et al., "Annexin A2 silencing inhibits invasion, migration, and tumorigenic potential of hepatoma cells," World Journal of Gastroenterology, vol. 19, no. 24, pp. 3792-3801, 2013.

[10] V. M. Díaz, M. Hurtado, T. M. Thomson, J. Reventós, and R. Paciucci, "Specific interaction of tissue-type plasminogen activator (t-PA) with annexin II on the membrane of pancreatic cancer cells activates plasminogen and promotes invasion in vitro," Gut, vol. 53, no. 7, pp. 993-1000, 2004.

[11] M. R. Sharma, L. Koltowski, R. T. Ownbey, G. P. Tuszynski, and M. C. Sharma, "Angiogenesis-associated protein annexin II in breast cancer: Selective expression in invasive breast cancer and contribution to tumor invasion and progression," Experimental and Molecular Pathology, vol. 81, no. 2, pp. 146-156, 2006.

[12] L. Tatenhorst, U. Rescher, V. Gerke, and W. Paulus, "Knockdown of annexin 2 decreases migration of human glioma cells in 
vitro," Neuropathology and Applied Neurobiology, vol. 32, no. 3, pp. 271-277, 2006.

[13] R. Duncan, B. Carpenter, L. C. Main, C. Telfer, and G. I. Murray, "Characterisation and protein expression profiling of annexins in colorectal cancer," British Journal of Cancer, vol. 98, no. 2, pp. 426-433, 2008.

[14] Q. Zhang, Z. Ye, Q. Yang, X. He, H. Wang, and Z. Zhao, "Upregulated expression of Annexin II is a prognostic marker for patients with gastric cancer," World Journal of Surgical Oncology, vol. 10, no. 1, p. 103, 2012.

[15] C. D. Paul, P. Mistriotis, and K. Konstantopoulos, "Cancer cell motility: Lessons from migration in confined spaces," Nature Reviews Cancer, vol. 17, no. 2, pp. 131-140, 2017.

[16] G. Chen, S.-M. Chen, X. Wang, X.-F. Ding, J. Ding, and L.-H. Meng, "Inhibition of chemokine (CXC motif) ligand 12/chemokine (CXC motif) receptor 4 axis (CXCL12/CXCR4)mediated cell migration by targeting mammalian target of rapamycin (mTOR) pathway in human gastric carcinoma cells," The Journal of Biological Chemistry, vol. 287, no. 15, pp. 1213212141, 2012.

[17] M. Xu, K. A. Bower, S. Wang et al., "Cyanidin-3-Glucoside inhibits ethanol-induced invasion of breast cancer cells overexpressing ErbB2," Molecular Cancer, vol. 9, no. 1, p. 285, 2010.

[18] X. Liu, J. Wu, D. Zhang et al., "Identification of Potential Key Genes Associated With the Pathogenesis and Prognosis of Gastric Cancer Based on Integrated Bioinformatics Analysis," Frontiers in Genetics, vol. 9, p. 265, 2018.

[19] N. Yang, L. Wang, J. Liu et al., "MicroRNA-206 regulates the epithelial-mesenchymal transition and inhibits the invasion and metastasis of prostate cancer cells by targeting Annexin A2," Oncology Letters, vol. 15, no. 6, pp. 8295-8302, 2018.

[20] A. Semov, M. J. Moreno, A. Onichtchenko et al., "Metastasisassociated protein S100A4 induces angiogenesis through interaction with annexin II and accelerated plasmin formation," The Journal of Biological Chemistry, vol. 280, no. 21, pp. 2083320841, 2005.

[21] T. J. Musholt, J. Hanack, C. Brehm, R. Von Wasielewski, and P. B. Musholt, "Searching for non-RET molecular alterations in medullary thyroid carcinoma: Expression analysis by mRNA differential display," World Journal of Surgery, vol. 29, no. 4, pp. 472-482, 2005.

[22] F. Han, S. Shrestha, H. Huang et al., "Expression of annexin II in gastric carcinoma and its role in gastric cancer metastasis," World Journal of Gastroenterology, vol. 23, no. 38, pp. 7009-7015, 2017.

[23] Y.-X. Wang, H. Lv, Z.-X. Li, C. Li, and X.-Y. Wu, "Effect of shRNA mediated down-regulation of Annexin A2 on biological behavior of human lung adencarcinoma cells A549," Pathology \& Oncology Research, vol. 18, no. 2, pp. 183-190, 2012.

[24] Z. Dong, M. Yao, H. Zhang et al., "Inhibition of Annexin A2 gene transcription is a promising molecular target for hepatoma cell proliferation and metastasis," Oncology Letters, vol. 7, no. 1, pp. 28-34, 2014.

[25] Y. Liu, H. Li, Z. Ban et al., "Annexin A2 inhibition suppresses ovarian cancer progression via regulating $\beta$-catenin/EMT," Oncology Reports, vol. 37, no. 6, pp. 3643-3650, 2017.

[26] F. Zhang, Z. Wang, J. Yuan, X. Wei, R. Tian, and R. Niu, "RNAi-mediated silencing of Anxa2 inhibits breast cancer cell proliferation by downregulating cyclin D1 in STAT3-dependent pathway," Breast Cancer Research and Treatment, vol. 153, no. 2, pp. 263-275, 2015.
[27] C.-Y. Wang, C.-L. Chen, Y.-L. Tseng et al., "Annexin A2 silencing induces G2 arrest of non-small cell lung cancer cells through p53-dependent and -independent mechanisms," The Journal of Biological Chemistry, vol. 287, no. 39, pp. 32512-32524, 2012.

[28] N. Tiwari, A. Gheldof, M. Tatari, and G. Christofori, "EMT as the ultimate survival mechanism of cancer cells," Seminars in Cancer Biology, vol. 22, no. 3, pp. 194-207, 2012.

[29] R. Xie, J. Liu, R. Wu et al., "The effect of lentivirus-mediated Annexin A2 silence on gene expression profiles of AGS gastric cancer cells," Modern Oncology, vol. 25, no. 3, pp. 362-367, 2017 (Chinese). 


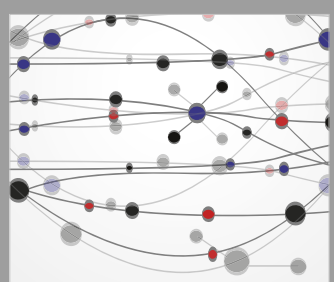

The Scientific World Journal
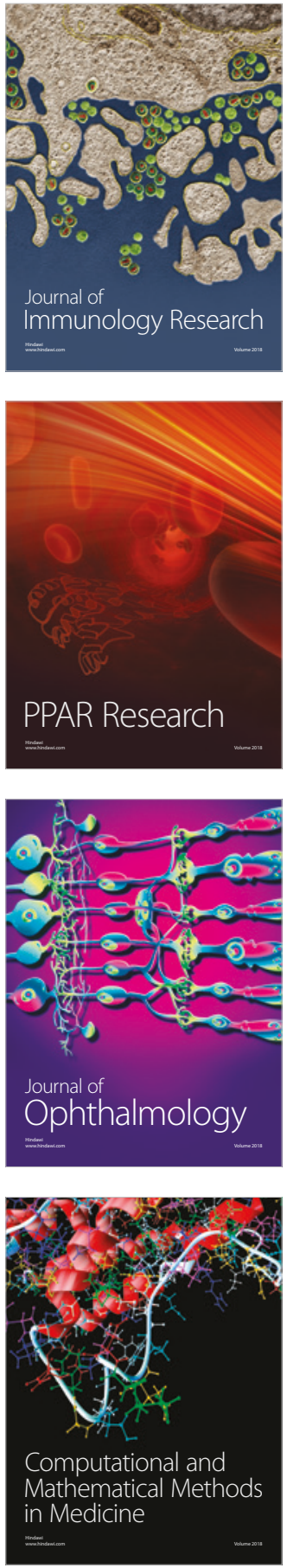

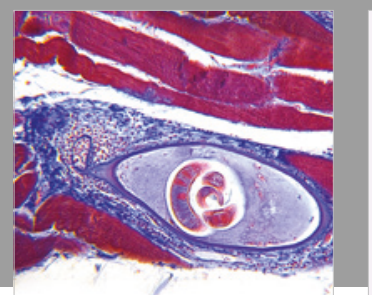

Gastroenterology Research and Practice

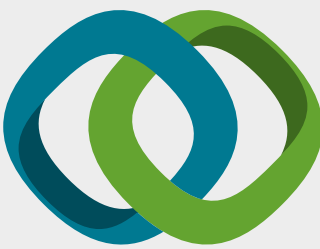

\section{Hindawi}

Submit your manuscripts at

www.hindawi.com
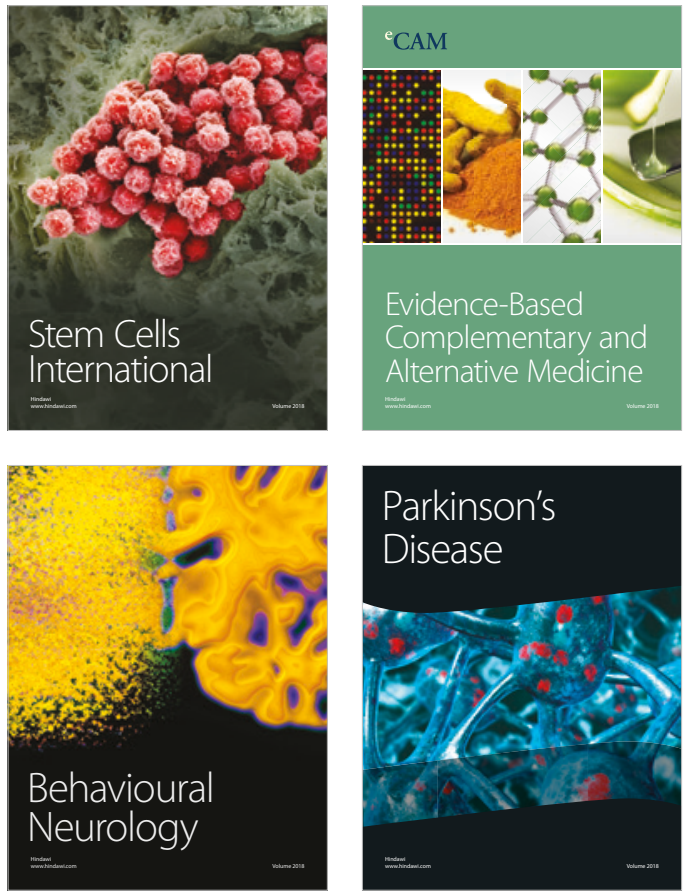

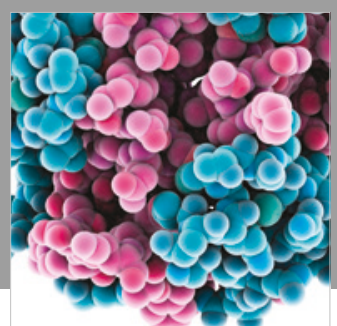

ournal of

Diabetes Research

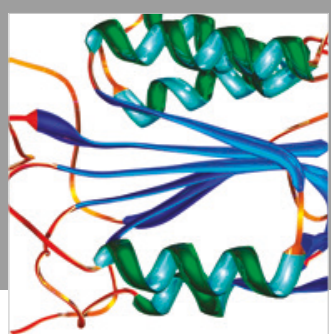

Disease Markers
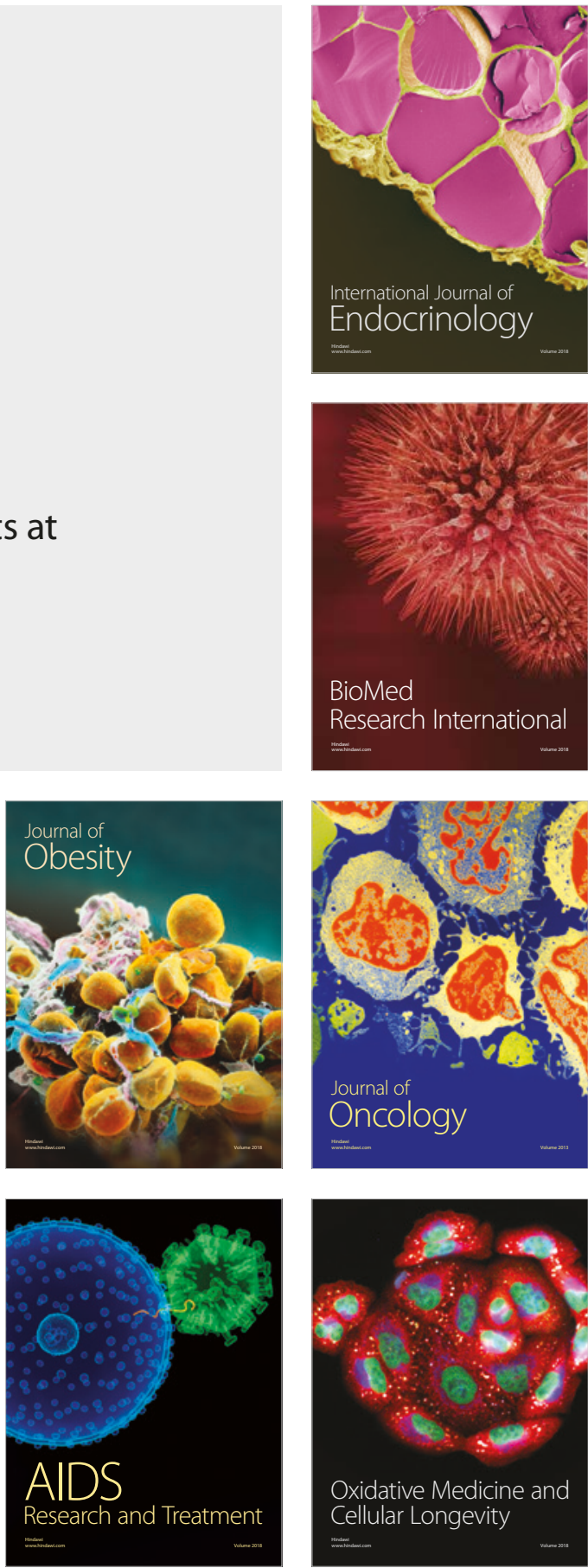